\title{
ON THE LEFT NUCLEUS OF A BRUCK RING
}

\section{R. L. SAN SOUCIE}

Let $F$ be a field of characteristic two and let $R$ be the set of all couples $(f, g)$ with $f$ and $g$ in $F$. Define equality and addition in $R$ componentwise, and define multiplication by

$$
(f, g)(h, k)=(f h+g \cdot k \theta, f k+g h),
$$

where $\theta$ is an additive endomorphism of $F . R$ is a right alternative ring which is alternative if and only if $k \theta=k m$, for all $k$ in $F$ and for some fixed $m$ in $F . R$ is a division ring if and only if, for every $f$ in $F$, the mapping $\mu(f)$, defined by

$$
x \mu(f)=x \theta+x f^{2}, \quad \text { all } x \text { in } F,
$$

is one-to-one of $F$ upon $F$. (See [2], ${ }^{1}$ Appendix.) R. H. Bruck has shown that fields $F$ having additive endomorphisms $\theta$ satisfying (1.2), and yet not right multiplications, actually exist. The author, in [1], has therefore called a not alternative, right alternative division ring $B$ (necessarily of characteristic two) a Bruck ring if it is two dimensional over some field $F$ and if multiplication in $B$ is given by (1.1). The field $F$ turns out to be the left nucleus of $B$ and, in all the examples given by Bruck, is a simple transcendental extension of a field of characteristic two, and thus imperfect.

In this note we give an elementary proof that this situation must of necessity occur:

THEOREM. The left nucleus of a Bruck ring is always an imperfect field.

Proof. If $B$ is a Bruck ring with left nucleus $F$, assume that $F$ is perfect. Let $\theta$ be the additive endomorphism of (1.1) and suppose that $x \theta=y, x \neq 0$ in $F$. Then $y \neq 0$ and $y=x z$, some $z$ in $F$. But then $z=v^{2}$, some $v$ in $F$, so that $x \theta=x v^{2}$ and $x \theta+x v^{2}=0$. However, $0 \theta+0 v^{2}$ $=0$ and thus the mapping $\mu(v)$, defined by (1.2), is not one-to-one of $F$ upon $F$. Hence $B$ is not a division ring, a contradiction. It must therefore be true that $F$ is imperfect, which is what we wanted to prove.

We remark that the following corollary answers a question raised by Kleinfeld in a letter to the author dated May, 1953 and mentioned by the author in [1] as an unsolved problem.

Corollary. There exist no finite Bruck rings.

Received by the editors December 20, 1955.

1 Numbers in brackets refer to the bibliography. 


\section{BIBLIOGRAPHY}

1. R. L. San Soucie, A characterization of a class of rings, Amer. J. Math. vol. 77 (1955) pp. 190-196.

2. - Right alternative division rings of characteristic two, Proc. Amer. Math. Soc. vol. 6 (1955) pp. 291-296.

UNIVERSITY OF OREGON

\section{THE EXISTENCE OF OUTER AUTOMORPHISMS OF SOME GROUPS}

\section{RIMHAK REE}

F. Haimo and E. Schenkman [1] raised the question: Does a nilpotent group $G$ always possess an outer automorphism? The answer is in the affirmative if $G$ is finite and nilpotent of class 2, as is seen from a Schenkman's [1] stronger result. The object of this note is to show that the answer is also in the affirmative for another family of nilpotent groups, namely the family of all finite $p$-groups $G$ of order greater than 2 such that $x^{p}=e$ for every element $x$ in $G$. Actually, our result is somewhat stronger:

THEOREM 1. Suppose that $G$ is a group every element of which is of order a divisor of a fixed integer $n>1$. If $G$ has a normal subgroup $N$ such that the factor group $G / N$ is cyclic of order $n$ and such that the intersection $N \cap Z$ of $N$ with the center $Z$ of $G$ contains an element $a_{0}$ of order $n$, then $G$ possesses an outer automorphism which induces identity automorphisms on both $N$ and $G / N$.

For the proof we need the following

Lемма. If elements $a, a_{0}, a_{1}, \cdots, a_{k}$ in a group $G$ are such that $a a_{0}=a_{0} a, a_{i} a_{j}=a_{j} a_{i}$ for $i, j=0,1, \cdots, k$ and such that

$$
a_{i} a a_{i}^{-1}=a a_{i-1}
$$

$$
(i=1,2, \cdots, k)
$$

then we have, for $r=1,2, \cdots$,

$$
\left(a a_{k}\right)^{r}=a^{r} a_{k}^{C_{r, 1}} a_{k-1}^{C_{r, 2}} \ldots a_{k-r+1}^{C_{r, r}},
$$

where we set $a_{-1}=a_{-2}=\cdots=e$. If, moreover, $a_{0}$ is of order $n$ and if $a_{i}^{n}=e$ for $i=1,2, \cdots, k$, then

Received by the editors December 27, 1955. 